\title{
ERP correlates of transposed-letter similarity effects: Are consonants processed differently from vowels?
}

\author{
Manuel Carreiras $^{\mathrm{a}, *}$, Marta Vergara $^{\mathrm{a}}$, Manuel Perea ${ }^{\mathrm{b}}$ \\ a Universidad de La Laguna, Spain \\ ${ }^{\mathrm{b}}$ Universitat de València, Spain
}

Received 9 February 2007; received in revised form 27 March 2007; accepted 18 April 2007

\begin{abstract}
Recent research has shown that pseudowords created by transposing letters are very effective for activating the lexical representation of their base words (e.g., relovution activates REVOLUTION). Furthermore, pseudoword transpositions of consonants are more similar to their corresponding base words than the transposition of vowels. We report one experiment using pseudowords created by the transposition of two consonants, two vowels, and their corresponding control conditions (i.e., the replacement of two consonants or two vowels) in a lexical decision task while Event Related Potentials (ERPs) were recorded. The results showed a modulation of the amplitude of the N400 component as a function of the type of pseudoword (transposed-letter versus replacement letter pseudowords), and this modulation was different for transposed consonants and vowels. These results suggest that consonants and vowels play a different role during word processing.
\end{abstract}

(C) 2007 Elsevier Ireland Ltd. All rights reserved.

Keywords: Visual-word recognition; Transposed-letters; ERPs; Consonants and vowels

When we read it is common to misread words like causal and casual. A growing number of studies have shown that, during the recognition of any given word, not only is the representation of the word itself activated, but also the representations of similarly spelled words ("neighbors"). Although the majority of "neighborhood" experiments have focused on one specific type of neighbor: one-letter different neighbors (e.g., trail and train), recent research has shown that transposed-letter neighbors may be even more perceptually similar to the target stimulus than oneletter different neighbors (trail and trial; [28,31]). Most notably, the presence of transposed-letter effects has critical implications for the choice of an input coding-scheme in visual-word recognition. Most current computational models of visual-word recognition $[7,13,20]$ assume that each letter is encoded in a different "letter-channel", and hence they cannot accommodate the presence of transposed-letter effects.

To overcome the limitations of a channel-specific codingscheme, a number of input coding-schemes have been proposed (SERIOL model [34]; SOLAR model [11]; open-bigram model

\footnotetext{
* Corresponding autor at: Departamento de Psicología Cognitiva, Universidad de La Laguna, 38205 Tenerife, Spain. Tel.: +34 922317515; fax: +34922317461

E-mail address: mcarreir@ull.es (M. Carreiras).
}

[14]; overlap model [12]). Although the basic mechanisms of how letter position is encoded differ across these models, they all predict that transposed-letter neighbors like casual and causal are perceptually very similar. There is one caveat, however: for simplicity's sake, these models assume that consonants and vowels are processed in the same way. As shown in the present paper, this assumption may be an oversimplification.

Recent transposed-letter experiments [28,27] have shown that consonants and vowels play a different role in visual-word recognition. In particular, Perea and Lupker [28] obtained masked priming effects for consonant transpositions (relovución-REVOLUCIÓN versus retosuciónREVOLUCIÓN), but not for vowel transpositions (reluvociónREVOLUCIÓN versus relavición-REVOLUCIÓN). Furthermore, these findings have been extended to a single-presentation lexical decision task [28,27]: transposed-letter pseudowords created by transposing two consonants (e.g., RELOVUCIÓN) produce a high number of "word" responses $(45 \%$ versus $5 \%$ of errors for the transposed-letter condition and its control, respectively), while effects for transposing two vowels (REVULOCIÓN) relative to the appropriate orthographic control are substantially smaller (25\% versus $5 \%$ of errors for the transposed-letter condition and its control, respectively). Thus, transposed-letter vowel pseudowords are also fairly sim- 
ilar to their base words, but they are simply less similar than transposed-letter consonant pseudowords. These results are consistent with claims that there may be some basic processing differences between vowels and consonants in the process of lexical access, and with findings from other paradigms (visual-word perception: [1,18]; speech perception: [2]; neuropsychology: $[4,9])$.

In particular, Cutler et al. [10] showed that, when allowed to change one phoneme to make a word from a pseudoword, participants more often alter a vowel than a consonant. Furthermore, statistical information about consonants, but not about vowels, appears to have a crucial role in the detection of words in the continuous speech stream $[3,22,26]$. Finally, a categorical distinction for vowels and consonants in the brain has been proposed by Caramazza et al. [4] (see also [8,9,21,33]) on the basis of neuropsychological evidence. This proposal is based on two aphasic patients who exhibit contrasting patterns of errors when producing vowels and consonants: one patient makes three times as many errors in vowels than in consonants, while the other patient makes five times as many errors in consonants than in vowels. This double dissociation has also been found in two other patients [8,21]. In addition, Carreiras and Price [5] found that changing vowels relative to consonants increased activation in the right middle temporal cortex, while changing consonants relative to vowels increased activation in right middle frontal cortex. Taken together, all these findings offer a strong case for a functional distinction between vowels and consonants.

The main goal of the present experiment is to examine transposed-letter effects in visual-word recognition, with specific attention to the role of consonants and vowels, by using electrophysiological measures. ERPs are voltage changes recorded from the scalp and extracted from the background electroencephalogram by averaging time-locked responses to stimuli onset. Of specific interest for our study is the N400 component, a negative deflection occurring around $400 \mathrm{~ms}$ after a word presentation that has been associated to lexical-semantic processing $[16,17]$. In particular, the amplitude of this negativity is an inverse function of the lexical frequency, of lexicality, and of orthographic neighborhood size. The amplitude of the N400 component is greater for low-frequency words than for high-frequency words, and for pseudowords than for words $[6,23]$. Furthermore, words embedded in a large neighborhood (in terms of "one-letter different" neighbors) generate a larger N400 component than words embedded in a sparse neighborhood. This is so because larger neighborhoods produce higher levels of activation, either at the level of form representations or at the level of semantic representation [16]. If transposedletter pseudowords generate more lexical activity-especially transposed-letter consonant pseudowords, they should produce a modulation of the amplitude in the same way as one-letter different neighbors.

In the present experiment, we examine whether transposedletter pseudowords created by transposing two consonants (or two vowels) are more competitive (in terms of increased amplitude of the N400 component, number of false positives, and longer latencies in the behavioral measures) than their corresponding orthographic controls in a lexical decision task. Highly wordlike pseudowords (e.g., RELOVUCIÓN) are expected to activate the lexical representation of their corresponding base words to a higher degree than orthographic controls (RETO$\left.S U C I O{ }^{N}\right)$, and therefore one would expect a strong tendency to misperceive them as words. Importantly, if transposed-letter pseudowords (RELOVUCIÓN) activate the lexical representation of their corresponding base words (REVOLUCIÓN) to a higher degree than the orthographic controls (RETOSUCIÓN), larger amplitudes in the $\mathrm{N} 400$ component will be expected for the transposed-letter pseudowords than for the replacement-letter pseudowords. Furthermore, on the basis of prior research, we would expect this effect to be larger for consonant transpositions than for vowel transpositions and also expect differences in topography between them.

Twenty-four (14 women) undergraduate students participated in the experiment in exchange for course credit. All of them were native Spanish speakers, with no history of neurological or psychiatric impairment, and with normal or corrected-to-normal vision. Ages ranged from 18 to 33 years (mean $=23.5$ years). All participants were right-handed, as assessed with a Spanish version of the Edinburgh Handedness Inventory [24].

The base words for the pseudoword targets were 240 Spanish words (mean word frequency per one million words in the [32], count: 23 , range: $1-147$; mean Coltheart's N: 0.5, range: 0-5; mean length in letters: 8.9, range: 7-11). For each base word, we created: (i) a transposed-letter pseudoword in which two nonadjacent consonants were switched (RELOVUCIÓN), (ii) a replacement-letter pseudoword in which the two critical consonants were replaced by others (RETOSUCIÓN), (iii) a transposed-letter pseudoword in which two nonadjacent vowels were switched (REVULOCIÓN), and (iv) a replacement-letter pseudoword in which the two critical vowels were replaced by others (REVALICIÓN). The transposed-letter pseudowords and their controls had, on average, 0.075 one-letter different neighbors (range 0-1) —all these neighbors were always verylow-frequency words. In all cases, the first syllable of the base word remained unchanged. An additional set of 240 words that were 7 to 11 letters long (mean frequency per million words: 31 , range: 4-251) was included for the purposes of the lexical decision task. Four lists of materials were constructed so that each pseudoword appeared once in each list, but each time in a different condition. Different participants were assigned randomly to each list.

Participants were seated comfortably in a darkened soundattenuated room. All stimuli were presented on a high-resolution monitor that was positioned at eye level $80-90 \mathrm{~cm}$ in front of the participant. The words were displayed in white uppercase Arial 24 against a dark-grey background. Participants performed a lexical decision task: they were instructed to press one of two buttons on the response pad to indicate whether the letter string was a legitimate Spanish word or not. A response button was positioned beneath each thumb. For half of the participants the right button was used to signal the "Yes" response and left button was assigned the "No" response. For the remaining participants the order was reversed. The sequence of events in each trial is described as follows. First, a fixation point ("+") appeared in the centre of the screen and remained there for $500 \mathrm{~ms}$. The 
fixation point was followed by a word or a pseudoword that remained there for $400 \mathrm{~ms}$. The inter-trial interval varied randomly between 1000 and $1300 \mathrm{~ms}$. The stimuli were presented in different random order for each participant.

Sixteen warm-up trials were provided at the beginning of the session and were repeated if necessary. Participants were also asked to avoid eye-movements and blinks during the interval starting from the fixation point until response was given. They were directed to favor accuracy over speed in their responses.

Scalp voltages were collected from $58 \mathrm{Ag} / \mathrm{AgCl}$ electrodes which were mounted in an elastic cap (ElectroCap International, Eaton, USA, 10-10 system). Linked earlobes were used as reference. Eye movements and blinks were monitored with six further electrodes providing bipolar recordings of the horizontal and vertical electrooculogram (EOG). Inter-electrode impedances were kept below $10 \mathrm{k} \Omega$. EEG was filtered with an analogue bandpass filter of $0.01-100 \mathrm{~Hz}$ and a digital $20 \mathrm{~Hz}$ low-pass filter was applied before analysis. The signals were sampled continuously throughout the experiment with a sampling rate of $250 \mathrm{~Hz}$.

Epochs of the EEG corresponding to $750 \mathrm{~ms}$ after word onset presentation were averaged and analyzed. Baseline correction was performed using the average EEG activity in the $100 \mathrm{~ms}$ preceding the onset of the target word as a reference signal value. Following baseline correction, epochs with simultaneous artifacts in at least 10 channels were rejected. In addition, trials that were not responded to correctly were not included in the analysis. This resulted in the exclusion of approximately $22.6 \%$ of the trials. Separate ERPs were formed for each of the experimental conditions, each of the participants and each of the electrode sites.

Six regions of interest were computed out of the 64 electrodes, each containing the mean of a group of electrodes. The regions were: left-anterior (F1,F3,F5,C1A,C3A,C5A), leftcentral (C1,C3,C5,C1P,C3P,TCP1), left-posterior (P1,P3,P5, $\mathrm{P} 1 \mathrm{P}, \mathrm{P} 3 \mathrm{P}, \mathrm{CB} 1)$, right-anterior (F2,F4,F6,C2A,C4A,C6A), rightcentral (C2,C4,C6,C2P,C4P,TCP2), right-posterior (P2,P4, P6,P2P,P4P,CB2).

Mean amplitudes were obtained for different time windows. For each window, a repeated-measures ANOVA was performed, including electrode regions (anterior, central and posterior), hemisphere (left/right) and the experimental variables as factors: type of similarity (transposed versus replaced) and type of transposed/replaced letter (consonants versus vowels). Where appropriate, critical values were adjusted using the Greenhouse-Geisser correction [15]. Effects for the electrode region factor or for the hemisphere factor will only be reported when they interact with the experimental manipulations.

The mean response times for correct responses and error rates of pseudowords are presented in Table 1. See also $d^{\prime}$ and $\log (\beta)$. Incorrect responses $(15.5 \%)$ were excluded from the latency analysis. In addition, to avoid the influence of outliers, reaction times less than $300 \mathrm{~ms}$ or greater than $1500 \mathrm{~ms}$ (less than $4.0 \%$ ) were excluded. ANOVAs based on subject mean response latencies and error rates to pseudoword targets were conducted based
Table 1

Mean lexical decision times (in ms) and standard deviations (in parentheses), percentage of errors, target detection sensitivity $\left(d^{\prime}\right)$ and response criterion $(\log (\beta))$ indices on pseudowords

\begin{tabular}{lccc}
\hline & Transposed-letter & Replacement-letter & Difference \\
\hline Consonants & & & \\
RT (S.D.) & $1041(124)$ & $917(116)$ & 124 \\
Errors & $30.4(14.4)$ & $6.7(5.7)$ & 23.8 \\
$d^{\prime}$ & 2.34 & 3.41 & -1.07 \\
$\log (\beta)$ & 0.29 & 1.56 & -1.27 \\
Vowels & & & \\
RT (S.D.) & $1022(126)$ & $911(123)$ & 111 \\
Errors & $18.8(13.0)$ & $6.0(5.5)$ & 12.8 \\
$d^{\prime}$ & 2.79 & 3.48 & -.69 \\
$\log (\beta)$ & 0.84 & 1.64 & -.8 \\
\hline
\end{tabular}

Note: The mean correct RT for word trials was $834 \mathrm{~ms}$ and the error rate was $4.2 \%$

on a 2 (Type of similarity: transposition versus replacement) $\times 2$ (Type of transposed/replaced letter: consonants versus vowels) design. All significant effects had $p<.05$.

Pseudowords created by transposing two letters were responded to $118 \mathrm{~ms}$ slower than pseudowords created by replacing those two letters, $F(1,23)=253.84$. Pseudowords created by transposing/replacing two consonants had slower latencies than pseudowords created by transposing/replacing two vowels, $F(1,23)=4.52$. The interaction between the two factors was not significant.

The ANOVA on the error data showed that there were significantly fewer errors to replacement-letter pseudowords than to transposed-letter pseudowords, $F(1,23)=73.33$. There were also more errors to pseudowords created by transposing/replacing two consonants than to pseudowords created by transposing/replacing two vowels, $F(1,23)=33.67$. The interaction of the two factors was significant, $F(1,23)=31.74$ : the transposition-letter effect was larger for the pseudowords created by transposing two consonants $(23.8 \% ; F(1,23)=95.15)$ than for the pseudowords created by transposing two vowels $(12.8 \% ; F(1,23)=31.83)$.

The ERP grand averages, time-locked to the onset of the target pseudowords and words showed, as usual, that the amplitude of the N400 component is larger for pseudowords than for words. More importantly, ERP grand averages time-locked to the onset of the four pseudowords, which are represented in Fig. 1 over six recording sites, showed clear differences between them in the N400 component in two windows 300-500 and 500-650. In the 300-500 ms window, the negativity is larger for the replaced conditions than for the transposed conditions. In contrast, in the $500-650 \mathrm{~ms}$ window, the negativity is larger for the transposed than for the replaced conditions, which is particularly clear in the case of consonants. Fig. 2 shows the topographical distribution over the scalp of all the above-described effects at a particular time within the $500-650 \mathrm{~ms}$ interval. Statistical analyses supported these observations. ANOVAs including the factors type of similarity (transposition versus replacement), type of transposed/replaced letters (consonants versus vowels), electrode regions (anterior, central, and posterior), and hemisphere 

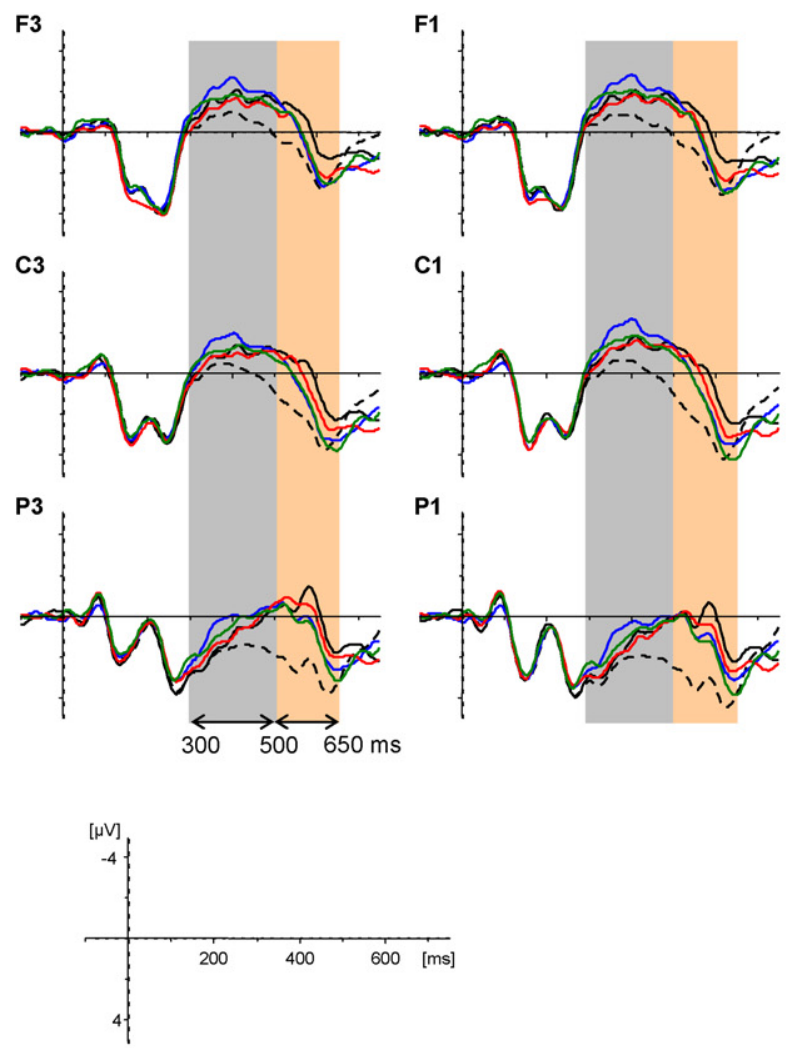
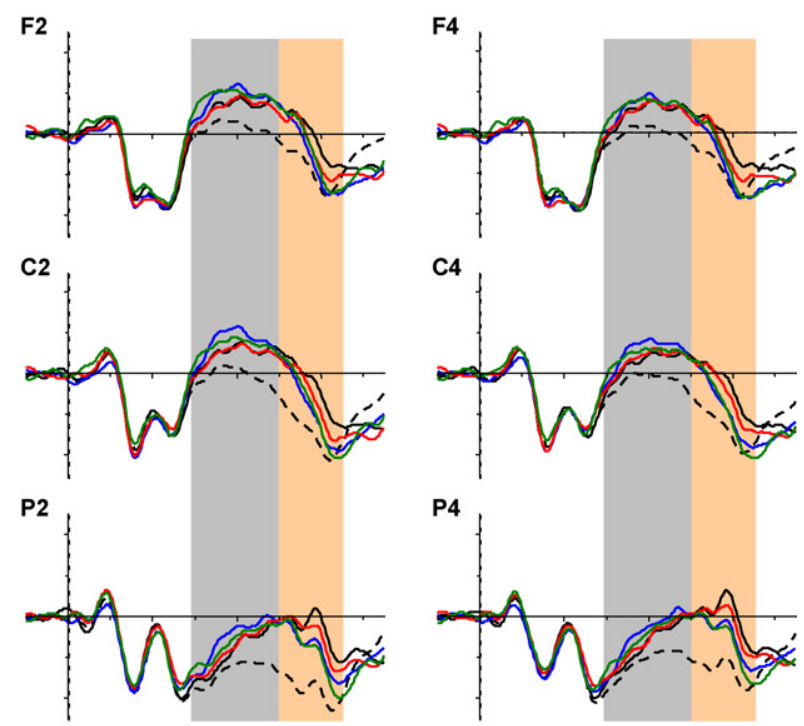

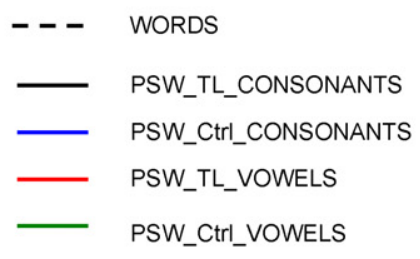

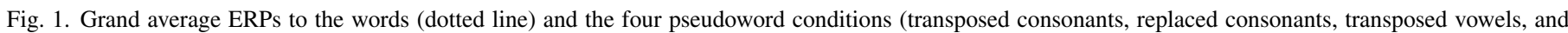

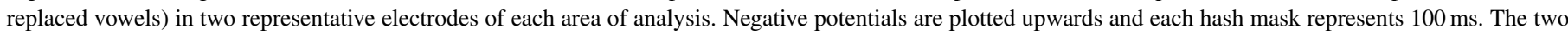
windows of analysis (300-500 and 500-650 ms) are highlighted by the vertical bars.

(left versus right) were performed separately for each of the two windows.

The ANOVA with the average values of the 300-500 ms time epoch showed a main effect of type of similarity $[F(1,23)=4.1$, $p<.05]$. The negativity amplitude was larger for the control replaced letters - than for the transposed conditions. None of the interactions type of similarity $\times$ type of letter $[F(1,23)=1.4]$, type of similarity $\times$ type of letter $\times$ hemisphere $[F(1,23)=1.1]$, type of similarity $\times$ type of letter $\times$ electrode $[F<1]$, or the fourway interaction $[F<1]$, was significant.

The ANOVA with the average values of the 500-650 ms time epoch showed a main effect of type of similarity $[F(1,23)=6.5$, $p<.01]$. Negativity amplitude was larger for the transposedletter conditions than for the replacement-letter conditions. However, this main effect was qualified by an interaction of type of similarity $\times$ type of letter $\times$ electrode regions $[F(2,46)=4.06$, $p<.05, \varepsilon=.568]$. In the anterior region, there was a significant effect of type of similarity for consonants $[F(1,23)=9.04$, $p<.01]$, but not for vowels $[F<1]$. In the middle region, the effect of type of similarity was significant both for consonants $[F(1,23)=4.5, p<.05]$ and for vowels $[F(1,23)=4.2, p<.05]$. Finally, in the posterior region, the effect of type of similarity was significant for vowels $[F(1,23)=4.5, p<.05]$, but not for consonants $[F(1,23)=1.6]$. The same pattern of effects was found
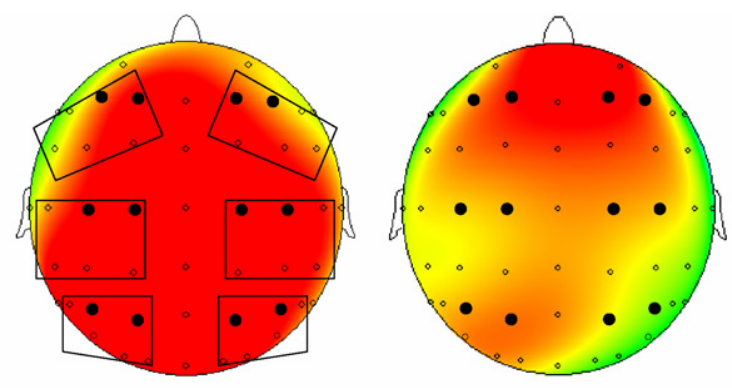

$300-500 \mathrm{~ms}$

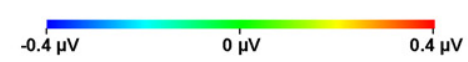

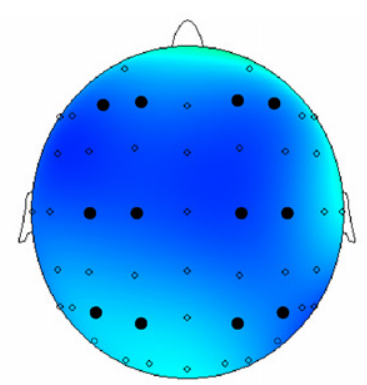

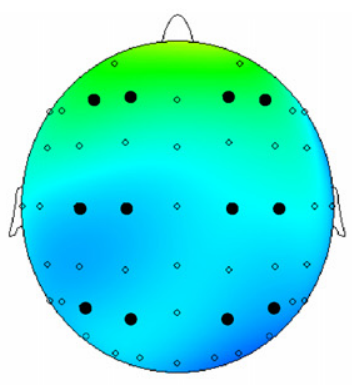

500-650 ms

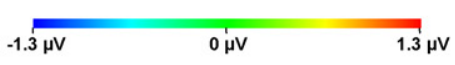

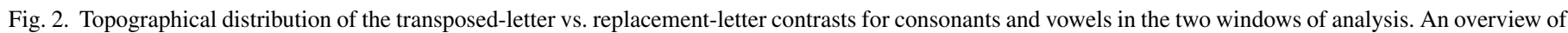

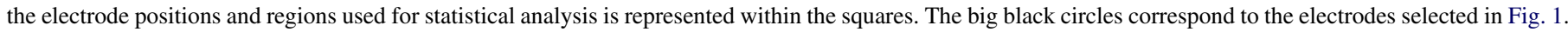


when the analyses were performed with a sample of the stimuli so that error rates were equated across conditions.

Consistent with previous research, transposed-letter pseudowords created by two transposed consonants activated their corresponding base words to a considerable degree [28,27]. This effect was quite dramatic in the false alarm rate, especially for consonant transpositions. Robust effects for transposing two vowels were also found both in the reaction times and error rates. Therefore, transposed-letter vowel pseudowords are also perceptually similar to their base words, but they are simply less similar than consonant transpositions.

More importantly, there were differences between consonants and vowels in the amplitude of the N400 component. In the first window (300-500 ms), the amplitude of the replacementletter condition was larger than that of the transposed-letter condition. In contrast, this tendency reverses in a later window (500-650 ms): the amplitude of the transposed-letter condition becomes larger than that of the replacement-letter condition. This late effect occurs for both consonants and vowels, but the topography of the effect differs: it is significant for consonants in the anterior and middle electrodes, whereas it is significant for vowels in the middle and posterior electrodes.

Thus, the present results suggest that, in the first window of the N400 component, transposed-letter pseudowords are treated almost as words and, hence, stimuli that are less "wordlike" - the replacement-letter conditions - produce larger amplitudes. This is why the amplitude of the replacement-letter pseudowords is enhanced relative to the transposed-letter pseudowords. However, this tendency immediately reverses because the transposed-letter pseudowords produce more lexical activity and become more competitive (i.e., "wordlike"). This pattern is consistent with the idea of an activation-verification process: an early activation stage and a later verification stage [25,29] (see [30] for an activation-verification model in silent reading). This interpretation is also consistent with the behavioral data [27-29].

The presence of transposed-letter similarity effects is consistent with the predictions of the recently-proposed coding schemes in visual-word recognition (SERIOL, SOLAR, openbigram, and overlap models). However, transposed-letter effects were different when the transposed letters were consonants than when they were vowels. In the above-cited models there is no difference between vowel and consonant processing, and hence, transposed-letter effects are posited to be of similar magnitude for vowel and consonant transpositions. One question for future research is whether these models can capture the observed effects by tweaking with the parameters or, whether, instead, they may need some more substantial modifications.

Interestingly, the differential pattern of data for consonants and vowels was noticeable in terms of the topographical distribution of the effects. This dissociation suggests functional differences for consonants and vowels in terms of cognitive processing and neural substrate [4,5]. Therefore, the data from patients, fMRI, as well as the ERP data converge on the idea that there may be some basic processing differences between vowels and consonants. Indeed, recent empirical evidence has also shown functional differences between consonants and vowels in speech perception and language acquisition $[3,22,26]$. Interestingly, Semitic languages attest the role of consonants in making lexical distinctions. In these languages, lexical roots are formed exclusively by consonants, whereas vowels are inserted to indicate morphological patterns [19]. Indeed, the fact that vowel processing during tasks that deal with visual-word recognition modulates activation in regions that have been also found to be modulated by prosody [5] is consistent with the proposal that vowels increase prosodic processing.

In sum, the present experiment has shown robust transposedletter effects in behavioral and electrophysiological measures. Importantly, the magnitude of these effects depends on whether the transposed-letters are vowels or consonants. Converging data from different paradigms suggest that there is differential processing for consonants and vowels. This functional distinction may reside in some differences in the neural representation. Further empirical and theoretical work is needed to shed more light on this important issue.

\section{Acknowledgements}

This research was partially supported by grants from the Spanish Ministry of Education and Science (SEJ2004-07680C02-02/PSIC, SEJ2005-05205/EDU, and SEJ2006-09238).

\section{References}

[1] I. Berent, C.A. Perfetti, A rose is a REEZ: the two cycles model of phonology assembly in reading English, Psychol. Rev. 102 (1995) 146-184.

[2] D. Boatman, C. Hall, M.H. Goldstein, R. Lesser, B. Gordon, Neuroperceptual differences in consonant and vowel discrimination: as revealed by direct cortical electrical interference, Cortex 33 (1997) 83-98.

[3] L.L. Bonatti, M. Peña, M. Nespor, J. Mehler, Linguistic constraints on statistical computations: the role of consonants and vowels in continuous speech processing, Psychol. Sci. 16 (2004) 451-459.

[4] A. Caramazza, D. Chialant, D. Capasso, G. Miceli, Separable processing of consonants and vowels, Nature 403 (2000) 428-430.

[5] M. Carreiras, C.J. Price, Brain activation for consonants and vowels, submitted for publication.

[6] M. Carreiras, M. Vergara, H. Barber, Early ERP effects of syllabic processing during visual word recognition, J. Cogn. Neurosci. 17 (2005) 1803-1817.

[7] M. Coltheart, K. Rastle, C. Perry, J. Ziegler, R. Langdon, DRC: A DualRoute Cascaded model of visual word recognition and reading aloud, Psychol. Rev. 108 (2001) 204-256.

[8] M. Cotelli, J. Abutalebi, M. Zorzi, S.F. Cappa, Vowels in the buffer: a case study of acquired dysgraphia with selective vowel substitutions, Cogn. Neuropsychol. 20 (2003) 99-114.

[9] R. Cubelli, A selective deficit for writing vowels in acquired dysgraphia, Nature 353 (1991) 258-260.

[10] A. Cutler, N. Sebastián-Gallés, O. Soler-Vilageliu, B. van Ooijen, Constraints of vowels and consonants on lexical selection: cross-linguistic comparisons, Memory Mem. Cogn. 28 (2000) 746-755.

[11] C.J. Davis. The Self-Organising Lexical Acquisition and Recognition (SOLAR) model of visual word recognition, Unpublished doctoral dissertation, University of New South Wales, 1999.

[12] P. Gómez, R. Ratcliff, M. Perea, A model of letter position coding: the overlap model, submitted for publication.

[13] J. Grainger, A.M. Jacobs, Orthographic processing in visual word recognition: a multiple read-out model, Psychol. Rev. 103 (1996) 518-565.

[14] J. Grainger, W.J.B. van Heuven, Modeling setter position coding in printed word perception, in: P. Bonin (Ed.), The Mental Lexicon, Nova Science, New York, 2003, pp. 1-23. 
[15] S. Greenhouse, S. Geisser, On methods in the analysis of profile data, Psychometrika 24 (1959) 95-112.

[16] P.J. Holcomb, J. Grainger, T. O'Rourke, An electrophysiological study of the effects of orthographic neighborhood size on printed word perception, J. Cogn. Neurosci. 14 (2002) 938-950.

[17] M. Kutas, K.D. Federmeier, Electrophysiology reveals semantic memory use in language comprehension, Trends Cogn. Sci. 4 (2000) 463-470.

[18] H.-W. Lee, K. Rayner, A. Pollatsek, The relative contribution of consonants and vowels to word identification during reading, J. Mem. Lang. 44 (2001) 189-205.

[19] J. McCarthy, Formal Problems in Semitic Phonology and Morphology, Garland, New York, 1985

[20] J.L. McClelland, D.E. Rumelhart, An interactive activation model of context effects in letter perception. Part 1. An account of basic findings, Psychol. Rev. 88 (1981) 375-407.

[21] G. Miceli, R. Capasso, B. Benvegnu, A. Caramazza, The categorical distinction of vowel and consonant representations: evidence from dysgraphia, Neurocase 10 (2004) 109-121.

[22] M. Nespor, M. Peña, J. Mehler, On the different roles of vowels and consonants in speech processing and language acquisition, Lingue e Linguaggio 2 (2003) 221-247.

[23] H.J. Neville, D.L. Mills, D.S. Lawson, Fractionating language: different neural subsystems with different sensitive periods, Cereb. Cortex 2 (1992) 244-258.

[24] R.C. Oldfield, The assessment and analysis of handedness: the Edinburgh inventory, Neuropsychologia 9 (1971) 97-113.
[25] K.R. Paap, S.L. Newsome, J.E. McDonald, R.W. Schvaneveldt, An activation-verification model for letter and word recognition: the word superiority effect, Psychol. Rev. 89 (1982) 573-594.

[26] M. Peña, L.L. Bonatti, M. Nespor, J. Mehler, Signal-driven computations in speech processing, Science 298 (2002) 604-607.

[27] M. Perea, M. Carreiras, Do transposed-letter effects occur at a syllabic level? Exp. Psychol. 53 (2006) 308-315.

[28] M. Perea, S.J. Lupker, Can CANISO activate CASINO? Transposed-letter similarity effects with nonadjacent letter positions, J. Mem. Lang. 51 (2004) 231-246.

[29] M. Perea, E. Rosa, C. Gómez, The frequency effect for pseudowords in the lexical decision task, Percept. Psychophys. 67 (2005) 301-314.

[30] E.D. Reichle, A. Pollatsek, D.L. Fisher, K. Rayner, Toward a model of eye movement control in reading, Psychol. Rev. 105 (1998) 125-157.

[31] S. Schoonbaert, J. Grainger, Letter position coding in printed word perception: effects of repeated and transposed letters, Lang. Cogn. Process. 19 (2004) 333-367.

[32] N. Sebastián-Gallés, M.A. Martí, M. Carreiras, F. Cuetos, LEXESP: una base de datos informatizada del español, Universitat de Barcelona, Barcelona, 2000.

[33] M.J. Tainturier, Rapp S B.C., Complex graphemes as functional spelling units: evidence from acquired dysgraphia, Neurocase 10 (2004) 122-131.

[34] C. Whitney, How the brain encodes the order of letters in a printed word: the SERIOL model and selective literature review, Psychon. Bull. Rev. 8 (2001) 221-243. 